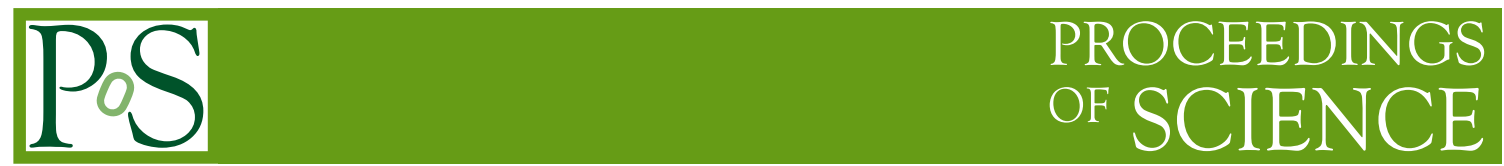

\title{
Cluster properties in noncommutative quantum field theory
}

Yuri Vernov*

Institute for Nuclear Research of Russian Academy of Sciences, Moscow, Russia E-mail: vernoveinr.ac.ru

M. N. Mnatsakanova

Skobeltsyn Institute of Nuclear Physics, Moscow, Russia

E-mail: mnatsaketheory.sinp.msu.ru

It has been proved that cluster properties of Wightman functions are valid in noncommutative quantum field theory as well as in the commutative case. This result is also valid in the space of arbitrary dimensions.

The XIXth International Workshop on High Energy Physics and Quantum Field Theory 8-15 September 2010

Golitsyno, Moscow, Russia

${ }^{*}$ Speaker. 


\section{Introduction}

Cluster properties of Wightman functions are very important in axiomatic approach in quantum field theory (QFT) [1, 2, 3]. Let us recall that cluster properties of Wightman functions imply that

$$
W\left(x_{1}, \ldots x_{k}, x_{k+1}+\lambda a, \ldots x_{n}+\lambda a\right) \rightarrow W\left(x_{1}, \ldots x_{k}\right) W\left(x_{k+1}, \ldots x_{n}\right),
$$

if $a^{2}=-1, \lambda \rightarrow \infty, W\left(x_{1}, \ldots x_{n}\right) \equiv\left\langle\Psi_{0}, \varphi\left(x_{1}\right) \ldots \varphi\left(x_{n}\right) \Psi_{0}\right\rangle$, where $\Psi_{0}$ is a vacuum vector.

Cluster properties are very natural consequence of Lorentz invariance. In fact, quantum field theory is defined by its Wightman functions [1]. Owing to Lorentz invariance quantum field operators are independent in points $x_{1}, \ldots x_{k}$ and points $x_{k+1}+\lambda a, \ldots x_{n}+\lambda a$. Thus it is natural to assume that

$$
W\left(x_{1}, \ldots x_{k}, x_{k+1}+\lambda a, \ldots x_{n}+\lambda a\right) \rightarrow W\left(x_{1}, \ldots x_{k}\right) W\left(x_{k+1}+\lambda a, \ldots x_{n}+\lambda a\right) .
$$

Rigorous proof of (1.2) one can find in [1, 2, 3]. In order to come to eq. (1.1) we have to take into account that Wightman functions are translation invariant.

In recent years noncommutative quantum field theory (NC QFT) attracts a great attention as very natural extension of the commutative theory to high energies (for review, see [4, 5]). Moreover, in some cases NC QFT is a low energy limit of string theory [6]. Thus it is very interesting to consider cluster properties in NC QFT. In this report we show that cluster properties are valid, if NC QFT is based on the following Heisenberg-like commutation relations between coordinates:

$$
\left[\hat{x}^{\mu}, \hat{x}^{v}\right]=i \theta^{\mu \nu}
$$

where $\theta^{\mu v}$ is a constant antisymmetric matrix.

It is known that NC QFT can be formulated in commutative space if the usual product of quantum field operators is substituted by the $\star$-product. Precisely, it implies that the usual product of the corresponding test functions is substituted by the $\star$-product. NC QFT is a physically important example of such a theory.

Let us point out that the class of theories, in which usual product is substituted by the $\star$ product, is wider than NC QFT as Heisenberg-like commutation relations imply the existence of $S O(1,1) \otimes S O(2)$ symmetry of the theory. Evidently, theories with the $\star$-product have not to possess such a symmetry in a general case.

Here we show that cluster properties are valid in NC QFT as well as in commutative QFT. Moreover, they are valid in any theory with the $\star$-product of test functions.

\section{Space-space Noncommutativity}

First let us consider the case, when time commutes with spatial variables. It is known that in this case there exists also one spatial variable, which commutes with all other variables [7]. Thus in this case we have two commutative variables, say $x^{0}$ and $x^{3}$ and two noncommutative ones: $x^{1}$ and $x^{2}$.

Let us stress that, actually, the number of noncommutative variables can be arbitrary. Let us point out that in the case of space-space noncommutativity there always exists one spatial variable, which commutes with all others irrespective of a number of noncommutative variables. 
We admit in correspondence with the standard QFT that Wightman functions are tempered distributions in respect with commutative variables. In fact, after integration over noncommutative variables we, actually, obtain two-dimensional commutative QFT. The cluster properties in this case exist on the same ground as in the four-dimensional theory. Thus we can use the standard methods and obtain that, actually, in this case in the theories with mass gap the difference between the left-hand member of the eq.(1.1) and its right-hand member falls more rapidly than $\lambda^{-n}$, where $n$ is an arbitrary positive number. In the theory with massless particles $n=1, a$ is two-dimensional vector: $a=\left\{a^{0}, a^{3}\right\}$.

\section{General Case}

The general case when all $\theta^{\mu v} \neq 0$ is much more nontrivial. Nevertheless we prove that eq.(1.1) is valid also in this case.

The crucial point in the derivation of eq.(1.1) is the fact that any test function $f_{i} \equiv f_{i}\left(x_{i}\right)$ belongs to the Gelfand-Shilov space $S^{\beta}$ with $\beta<1 / 2$ [8]. Precisely it was shown in our paper [9] that if $f_{i} \in S^{\beta}$ with $\beta<1 / 2$, then

$$
\begin{gathered}
f_{i} \star f_{i+1} \equiv \exp \left(\frac{i}{2} \theta^{\mu \nu} \frac{\partial}{\partial x_{i}^{\mu}} \frac{\partial}{\partial x_{i+1}^{v}}\right) f_{i}\left(x_{i}\right) f_{i+1}\left(x_{i+1}\right)= \\
\sum_{n=0}^{\infty} \frac{1}{n !}\left(\frac{i}{2} \theta^{\mu \nu} \frac{\partial}{\partial x_{i}^{\mu}} \frac{\partial}{\partial x_{i+1}^{v}}\right)^{n} f_{i}\left(x_{i}\right) f_{i+1}\left(x_{i+1}\right)
\end{gathered}
$$

converges. The similar result was obtained also in the paper of Soloviev [10].

If $f_{i} \star f_{i+1}$ converges $\forall i$, then $f_{1} \star \ldots \star f_{n}$ converges as well if $f_{i} \in S^{\beta}$ with $\beta<1 / 2$. Moreover this function belongs to the space $S^{\beta}$ with the same $\beta$.

Let us remind that in the simplest case of one variable $f(x)$ belongs to the space $S^{\beta}$ if

$$
\left|x^{k} \frac{d^{q} f(x)}{d x^{q}}\right| \leq C_{k} B^{q} q^{q \beta}, \quad-\infty<x<\infty, \quad k, q \in \mathbb{N},
$$

where the constants $C_{k}$ and $B$ depend on the function $f(x)$. In fact, for convergence of the series (3.1) it is sufficient to use the inequality (3.2) only at $k=0$. The extension of the last inequality on the case of multivariable function is straightforward.

Convergence of the series (3.1) implies that

$$
f_{1}\left(x_{1}\right) \star \cdots \star f_{n}\left(x_{n}\right)=f_{1}^{N}\left(x_{1}\right) \star \cdots \star f_{n}^{N}\left(x_{n}\right)+\varepsilon(N), \varepsilon(N) \rightarrow 0, \quad \text { if } \quad N \rightarrow \infty,
$$

where

$$
f_{i}^{N}\left(x_{i}\right) \star f_{i+1}^{N}\left(x_{i+1}\right)=\sum_{n=0}^{N} \frac{1}{n !}\left(\frac{i}{2} \theta^{\mu \nu} \frac{\partial}{\partial x_{i}^{\mu}} \frac{\partial}{\partial x_{i+1}^{v}}\right)^{n} f_{i}\left(x_{i}\right) f_{i+1}\left(x_{i+1}\right) .
$$

Let us stress that as $B$ in inequality (3.2) depends on function $f(x)$, but does not depend on $x$, thus estimation (3.1) is the same for every $x_{i}$.

Now let us point out that as $f_{i}^{N}\left(x_{i}\right) \star f_{i+1}^{N}$ contains only a finite numbers of derivatives, then the corresponding generalized functions are tempered distributions. 
Thus at any $N$ we have cluster properties. This fact gives us the possibility to prove cluster properties in the general case. Let us point out that $W\left(x_{1}, \ldots x_{n}\right)$ is a functional in the space of functions $f_{1}\left(x_{1}\right) \star \cdots \star f_{n}\left(x_{n}\right)$. Actually, on the one hand, for arbitrary $\lambda$

$$
W\left(x_{1}, \ldots x_{k}, x_{k+1}+\lambda a, \ldots x_{n}+\lambda a\right) \rightarrow W^{N}\left(x_{1}, \ldots x_{k}, x_{k+1}+\lambda a, \ldots x_{n}+\lambda a\right)+\varepsilon(N),
$$

where $\varepsilon(N) \rightarrow 0$, if $N \rightarrow \infty$. Here $a$ is a four-dimensional vector. On the other hand, at any $N$

$$
W^{N}\left(x_{1}, \ldots x_{k}, x_{k+1}+\lambda a, \ldots x_{n}+\lambda a\right)=W^{N}\left(x_{1}, \ldots x_{k}\right) W^{N}\left(x_{k+1}, \ldots x_{n}\right)+\varepsilon(\lambda),
$$

$\varepsilon(\lambda) \rightarrow 0$, if $\lambda \rightarrow \infty$. Combining the eqs. (3.4) and (3.5), we come to eq. (1.1):

$$
W\left(x_{1}, \ldots x_{k}, x_{k+1}+\lambda a, \ldots x_{n}+\lambda a\right) \rightarrow W\left(x_{1}, \ldots x_{k}\right) W\left(x_{k+1}, \ldots x_{n}\right) .
$$

Thus the cluster properties of Wightman functions are proved in any theory, in which the usual product of test functions is substituted by the $\star$-product. Physically important example of such a theory is NC QFT.

The most important consequence of cluster properties is the uniqueness of vacuum state. Thus we have proved that the uniqueness of vacuum state takes place in NC QFT as well as in the commutative theory.

\section{Conclusions}

It has been proved that cluster properties of Wightman functions are valid in any theory, in which usual product between test functions is substituted by the $\star$-product, as well as in the standard case. The important example of such a theory is noncommutative quantum field theory.

Let us stress that as in the theory under consideration the assertion that test functions belong to the Gelfand-Shilov space $S^{\beta}, \beta<1 / 2$ does not depend on the dimension of the underlying space, all our results are valid in the space of arbitrary dimensions.

\section{References}

[1] N. N. Bogoliubov, A. A. Logunov and I. T. Todorov, Introduction to Axiomatic Quantum Field Theory, Benjamin, Reading, Mass 1975.

[2] R. F. Streater and A. S. Wightman, PCT, Spin and Statistics and All That, Benjamin, NewYork 1964.

[3] R. Jost, The general theory of quantized fields, A.M.S., Providence, RI 1965.

[4] M. R. Douglas and N. A. Nekrasov, Noncommutative field theory, Rev. Mod. Phys., 73977 (2001), [hep-th/0106048].

[5] R. J. Szabo, Quantum field theory on noncommutative spaces, Phys. Rept., 378207 (2003), [hep-th/0109162].

[6] N. Seiberg, E. Witten, String theory and noncommutative geometry, JHEP, 990932 (1999), [hep-th/9908142].

[7] L. Álvarez-Gaumé, J. L. F. Barbon and R. Zwicky, Remarks on Time-Space Noncommutative Field Theories, JHEP 0105057 (2001), [hep-th/ 010306 9]. 
[8] I. Gel'fand and G. Shilov Generalized Functions V.2. Chapter IV, Academic Press Inc., New York 1968.

[9] M. Chaichian, M. N. Mnatsakanova, A. Tureanu and Yu. S. Vernov, Test Functions Space in Noncommutative Quantum Field Theory, JHEP 09 (2008) 125, [hep-th/ $0706.1712 \mathrm{v} 1]$.

[10] M. A. Soloviev, Star product algebras of test functions, Theor. Math. Phys. 153 (2007) 1351, [math-ph/0708.0811]. 